\title{
Effect of team training and monitoring on the rate of failed mid and low cavity vacuum extraction: a hospital based intervention study
}

\author{
Kristina Pettersson ${ }^{1,2^{*}}$ (D), Magnus Westgren ${ }^{2}$, Rebecca Götze-Eriksson ${ }^{1}$ and Gunilla Ajne ${ }^{1,2}$
}

\begin{abstract}
Background: Clinical team training has been advocated as a means to improve delivery care, and failed extractions is a suggested variable for clinical audit in instrumental vaginal delivery.

Other activities may also have intended or unintended effects on care processes or outcomes.

Methods: We retrospectively observed 1074 mid and low vacuum extraction deliveries during three time periods (prevalence periods): Baseline (period 0), implemented team training (period 1 and 2) and monitoring of traction force during vacuum extraction (period 2). Our primary outcome was failed extraction followed by emergency cesarean section or obstetric forceps delivery.
\end{abstract}

Results: The prevalence proportion (relative risk) of failed extraction decreased significantly after implementation of team training, from 19\% (period 0) to $8 \%$ (period 1), corresponding to a relative risk of 0.48 [0.26-0.87]. The secondary procedural outcome complicated delivery (duration $>15 \mathrm{~min}$ or number of pulls $>6$, or cup detachment $>1$ ) was decreased in period 2 compared to period 1, RR 0.42 [0.23-0.76]. Secondary clinical (neonatal) outcome were not affected.

Conclusion: Clinically based educational efforts and increased monitoring improved procedural outcome without improving neonatal outcome. The study design has inherent limitations in making causal inference.

Keywords: Vacuum extraction, Failed extraction, Team training, Hawthorne effect, Monitoring

\section{Background}

Good practice guidelines for obstetric care [1-3] has become increasingly important following reports on poor judgement as a cause of perinatal mortality and severe morbidity [4-7]. Furthermore, in order to meet the possible negative consequences of increasing rates of cesarean section [8], the argument for safe operative vaginal delivery is crucial. In Sweden, a national initiative of educational and policy interventions has been introduced to generally improve delivery care [9], including vacuum extraction delivery, but evaluating these policy changes has proven to be a significant challenge, and the effects on perinatal asphyxia

\footnotetext{
* Correspondence: kristina.a.pettersson@sll.se

${ }^{1}$ Department of Obstetrics and Gynecology, Karolinska University Hospital at Huddinge, K57, 14186 Stockholm, Sweden

${ }^{2}$ Clintec, Karolinska Institute, Stockholm, Sweden
}

is ambiguous [10]. Some studies, however, have shown measurable results from educational endeavors, such as a $50 \%$ decreased risk of obstetric anal sphincter injuries in Norway [11]; a decreased prevalence of severe asphyxia following the implementation of a national educational program in Australia [12], and a simultaneous decrease in emergency cesarean section and operative vaginal delivery in Sweden [13]. Some of the components of effective team training are in-house-setting, multi-professional teams, realistic training tools, and regular recurrence [14]. Suitably designed educational programs can potentially increase quality and enhance safety, but their effects require evaluation.

One possible quality and safety indicator is failed extraction, since this has been identified as a risk factor for adverse perinatal outcome [15-19], and recommended

(c) The Author(s). 2019 Open Access This article is distributed under the terms of the Creative Commons Attribution 4.0 International License (http://creativecommons.org/licenses/by/4.0/), which permits unrestricted use, distribution, and reproduction in any medium, provided you give appropriate credit to the original author(s) and the source, provide a link to the Creative Commons license, and indicate if changes were made. The Creative Commons Public Domain Dedication waiver (http://creativecommons.org/publicdomain/zero/1.0/) applies to the data made available in this article, unless otherwise stated. 
as audible standards by Royal College of Obstetricians and Gynaecologists [1]. A further risk increase seems to occur when multiple modes are needed, that is vacuum and forceps attempt preceding emergency cesarean section $[18,20]$.

This pre-post intervention study aimed to observe measurable effects on the safety of vacuum extraction deliveries when introducing team training and increased monitoring aimed at vacuum extraction. We compared three time periods characterized by different clinic-based activities that might influence how vacuum extraction is performed: Baseline (period 0), implemented team training (period 1 and 2) and monitoring of traction force during vacuum extraction (period 2). These activities are presented in more detail in the Material and methods section. Our primary hypothesis was a decreased failed extraction rate following team training (period 1), due to an increased adherence to guidelines in the delivery team, including obstetricians' technique. In period 2, after the additional introduction of objective traction force measurement, we wanted to observe any alteration in the failed extraction rate.

\section{Methods}

We retrospectively included all women at Karolinska University hospital in Huddinge, Sweden who underwent delivery by complete or attempted vacuum extraction at fetal head station low or mid during three time periods, $N=$ 1074. Period 0: 2007-2008; Period 1: 2011-2012; Period 2: 2013-2014. (2009-2010 are excluded as they were transition years when the educational program was in a start-up phase). See Fig. 1 for timeline of exposure. The design is conceptually equivalent to a cross-sectional study with three prevalence periods. We identified the cohorts retrospectively in the electronic medical records system (Obstetrix( ). Each individual vacuum extraction protocol was examined to confirm and separately classify mid vs low deliveries according to American and British guidelines [1, 2], since this distinction of low vs mid is not available in Swedish registers. Vacuum extractions in Sweden are nearly exclusively carried out by doctors, and for non-outlet extractions metal cups are often used, nearly exclusively Bird metal cup size $50 \mathrm{~mm}$. In this material only one non-outlet extraction was carried out by a midwife. All extractions reported as outlet extractions were excluded, since clinical experience and our previous data indicate that outlet extractions seldom lead to failed extractions or other complications [21]. Preterm delivery ( $<36$ full weeks of gestation) and multiple pregnancies were excluded. See Additional file 1 for flow chart. Data collection of clinical variables was carried out by two experienced medical doctors (residents) in obstetrics and gynecology. In addition, we identified all cases with emergency cesarean section at fully dilated cervix by scrutinizing the partogram and medical record text of all emergency cesarean section records. A description is presented of the overall registered rates of vacuum extraction, the proportion of non-outlet (low and mid) extractions, and emergency cesarean sections at the clinic during the time periods.

\section{Exposure (interventions)}

Period 0 constitutes a conceptual null exposure, that is the vacuum extraction management prior to implementing active structured measures to improve delivery care. These structured improvement actions therefore form the exposure or intervention in period 1 , and are described in detail in Additional file 2. In summary, they consist of a time out check list for vacuum extractions, annually recurrent clinical setting multi-professional team training based on vacuum extraction cases, and education regarding risk factors for complicated extractions. The team training sessions are led by a senior consultant and experienced midwife, and set in a delivery room using a mock pelvis and live actor from the staff, with direct feed back from a peer group rather than video recordings. The exposure or intervention of period 2 is the research based introduction of an electronical extraction handle which objectively measures and records the magnitude and duration of traction force employed in metal cup extractions.

All term, singleton deliveries were eligible, and there was no feedback on traction force to the obstetrician.

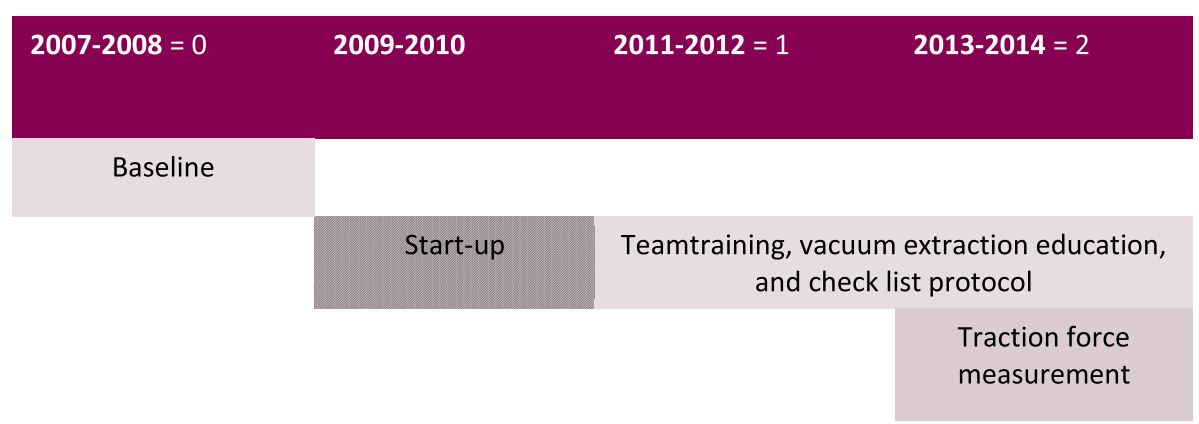

Fig. 1 Timeline of exposure periods 
These measurements infer an unintended but foreseen monitoring of individual performance. Both exposures may also be afflicted with a general highlighting effect that we believe is inevitable when all personnel are participating in a specific project, a concept known as the Hawthorne effect [22]. The equipment and results of traction force measurement have been described in detail by the authors elsewhere [21, 23].

\section{Clinical characteristics}

Maternal variables included age (years); short stature $(<1.55 \mathrm{~m})$; obesity (Body mass index $\geq 30$ ) and parity $(0 / 1)$. Obstetric variables included gestational age (days); use of synthetic oxytocin $(\mathrm{y} / \mathrm{n})$; epidural analgesia $(y / n)$; duration of first and second stage (minutes); fetal head station identified in the vacuum extraction protocol (low vs mid); fetal head position (occiput anterior or non-occiput anterior); indication of the procedure (dystocia or fetal distress, where dystocia included maternal fatigue and prophylactic); large for gestational age $\geq 4500 \mathrm{~g}$. A registered double diagnosis of indication was scrutinized by the data collectors, requiring either a non-reassuring cardiotocogram or an elevated scalp lactate to be indexed as fetal distress); duration of the extraction (minutes from beginning of extraction until final cup removal).

\section{Outcome variables}

The primary outcome variable, failed extraction, was identified in the medical records. Failed vacuum was defined as an attempt at extraction followed by forceps, emergency cesarean section, or both. The procedural secondary outcomes were failed extraction with more than one sequential mode of delivery (followed by both forceps and cesarean section), as well as complicated extractions, defined as one or more of the following: more than 15 min duration; more than six pulls, or more than one cup detachment. Clinical secondary outcome included shoulder dystocia $(\mathrm{y} / \mathrm{n})$; asphyxia $(\mathrm{pH}<7,0 \mathrm{y} / \mathrm{n})$; APGAR score $<7$ at $5 \mathrm{~min}(\mathrm{y} / \mathrm{n})$; admission to neonatal intensive care unit $(\mathrm{y} / \mathrm{n})$ and anal sphincter injury $(\mathrm{y} / \mathrm{n})$.

\section{Statistical analyses}

Clinical characteristics were analyzed using descriptive frequency measures and hypothesis tests as applicable: Mean (standard deviation) and t-test for normally distributed numerical variables, median (interquartile range) Rank sum and Kruskal-Wallis for skewed continuous data, and $\mathrm{Chi}^{2}$ for categorical data. As advised for analytical cross-sectional studies, Poisson regression was used for multivariate analysis of association between the exposure(s) and outcome, providing an approximation of a relative risk estimate based on prevalence proportions $[24,25]$. $P$-values in the multivariate analyses were Bonferroni corrected due to multiple testing. The primary and secondary procedural outcomes were adjusted by year of birth. No other available variables were identified as confounders. We have not performed repeat measurement calculations as generalized estimating equation or mixed models effect, as the recurring individuals constituted only $1 \%$ of the study sample. Confidence intervals regarding differences involving total delivery numbers in the sample (all vacuum extractions, emergency cesarean section) were calculated by hand, since they are based on group data rather than individuals.

\section{Missing data}

There was no missing data in primary outcome. The secondary procedural outcome complicated extraction was missing in $3 \%$. Maternal body mass index was the only clinical variable missing in more than $1 \%$ : (7.5\%). Since these were not primary variables, we decided not to handle this missing data further.

\section{Results}

The proportion of failed extractions among low and mid extractions was $12 \%$, and sequential instruments were used in $1.5 \%$. In approximately half of the cases, the indication for vacuum extraction was dystocia. More than half of the extractions were carried out at mid cavity fetal head station.

Maternal and obstetric clinical characteristics are shown in Table 1. Epidural and oxytocin use, parity, occiput anterior position, obesity and large for gestational age showed no significant differences between the three exposure periods. All other independent variables displayed some degree of difference throughout the study periods.

Primary and secondary procedural outcome is shown in Table 2. The primary outcome failed vacuum extraction decreased significantly in period 1 compared to period 0 , adjusted relative risk 0.48 . A significant decrease in complicated extractions was seen between period 1 and 2, adjusted relative risk 0.42 .

The secondary outcome multiple sequential modes of delivery could not be calculated due to few cases: thirteen cases in period 0 , and two cases in period 1 and 2 respectively. Secondary clinical outcome showed no significant differences (Additional file 3: Table S1).

The distribution of operative modes of delivery in the study sample is shown in Fig. 2. The total rate of vacuum extraction has decreased; period 0 9.7\%; period 1 $8.8 \%$; period $26.5 \%(p<0.05)$. The proportion of low and mid extractions (as compared to outlet) increased markedly throughout the periods: period $036 \%$; period 1 $45 \%$; period $260 \%(\mathrm{p}<0.05)$. There was no significant increase in emergency cesarean sections between the 
Table 1 Clinical characteristics

\begin{tabular}{|c|c|c|c|c|c|}
\hline & Period $0(n=328)$ & Period $1(n=370)$ & Period $2(n=376)$ & All $N=1074$ & \\
\hline Age, years ${ }^{\S}$ & $31(5)$ & $31(5)$ & $30(5)$ & $31(5)$ & $\begin{array}{l}p^{a} N S \\
p^{b}<0.05 \\
p^{c} N S\end{array}$ \\
\hline $\mathrm{BMI} \geq 30$ & $24(9)$ & $42(12)$ & $37(10)$ & $103(10)$ & NS \\
\hline Height $\mathrm{cm} \leq 1,55$ & $9(3)$ & $27(7)$ & $20(5)$ & $56(6)$ & $\begin{array}{l}p^{a}<0.05 \\
p^{b} N S \\
p^{c} N S\end{array}$ \\
\hline Nulliparous & $264(80)$ & $297(80)$ & $294(78)$ & $855(80)$ & NS \\
\hline GL, days $\#$ & $283(276-289)$ & $283(276-288)$ & $280(273-287)$ & $281(275-287)$ & $\begin{array}{l}p^{a} N S \\
p^{b}<0.01 \\
p^{c}<0.01\end{array}$ \\
\hline $\begin{array}{l}\text { Duration 1st stage min } \\
\text { median\# }\end{array}$ & $480(300-690)$ & $540(360-750)$ & $480(300-660)$ & $510(330-690)$ & $\begin{array}{l}p^{a}<0.05 \\
p^{b}<0.01 \\
p^{c} N S\end{array}$ \\
\hline $\begin{array}{l}\text { Duration 2nd stage min } \\
\text { median }^{\#}\end{array}$ & $120(60-210)$ & $150(60-210)$ & $165(90-230)$ & $150(60-210)$ & $\begin{array}{l}p^{a}<0.05 \\
p^{b} N S \\
p^{c}<0.01\end{array}$ \\
\hline Epidural & $245(75)$ & $283(77)$ & $297(79)$ & $825(77)$ & NS \\
\hline Oxytocin & 304 (93) & $348(94)$ & 354 (94) & $1006(94)$ & NS \\
\hline Indication dystocia & $168(51)$ & $227(62)$ & $202(54)$ & $597(56)$ & $\begin{array}{l}p^{a}<0.01 \\
p^{b}<0.05 \\
p^{c} N S\end{array}$ \\
\hline Fetal head station mid & $190(58)$ & $248(68)$ & $231(61)$ & $678(62)$ & $\begin{array}{l}p^{a}<0.01 \\
p^{b} N S \\
p^{c} N S\end{array}$ \\
\hline Birthweight $\geq 4500 \mathrm{~g}$ & $10(3)$ & $15(4)$ & $9(2)$ & $34(3)$ & NS \\
\hline Position non-OAP & $37(11)$ & $52(14)$ & $54(15)$ & $143(13)$ & NS \\
\hline Duration $>15 \mathrm{~min}^{*}$ & $15(5)$ & $44(12)$ & $21(6)$ & $80(7)$ & $\begin{array}{l}p^{a}<0.01 \\
p^{b}<0.01 \\
p^{c} N S\end{array}$ \\
\hline Nr pulls $>6^{*}$ & $34(11)$ & $43(12)$ & $23(6)$ & $100(10)$ & $\begin{array}{l}\mathrm{p}^{\mathrm{a}} \mathrm{NS} \\
\mathrm{p}^{\mathrm{b}}<0.01 \\
\mathrm{p}^{c}<0.05\end{array}$ \\
\hline Cup detachment $>1^{*}$ & $24(7)$ & $15(4)$ & $10(3)$ & $49(5)$ & $\begin{array}{l}\mathrm{p}^{\mathrm{a}} \mathrm{NS} \\
\mathrm{p}^{\mathrm{b}} \mathrm{NS} \\
\mathrm{p}^{c}<0.01\end{array}$ \\
\hline
\end{tabular}

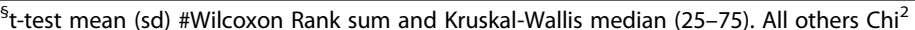

$\mathrm{a}=$ period 0 vs $1, \mathrm{~b}=$ period 1 vs $2, \mathrm{c}=$ period 0 vs 2

*Variables part of the composite secondary outcome variable complicated extraction

first two periods, but from period 1 to period 2 the rate increased by $1.3 \%$ units $(\mathrm{p}<0.05)$. The rate of emergency cesarean section without prior attempt of vacuum extraction at fully dilated cervix and engaged fetal head showed a non-significant increasing trend: period 0 : 2.1\%; period 1: $2.7 \%$; period 2: $3.4 \%$.

\section{Discussion}

\section{Main findings}

The main finding of this study is the decreased frequency of failed extractions after introduction of clinical team training, and a decreased risk of complicated extraction at the additional introduction of monitoring through traction force measurement. The total frequency of vacuum extraction also decreased during the whole study period, while the proportion of emergency cesarean section was increased.

\section{Interpretation (in the light of other evidence)}

To the best of our knowledge, no previous study has specifically investigated the effect of clinical team training or monitoring traction force on failed extraction rates. However, previous studies have shown positive effects of team training [14], and educational efforts have been found to decrease the frequency of anal sphincter injury and asphyxia, and to increase rates of normal delivery [11-13].

The subsiding effect on the failure rate after period I complicates the interpretation; does the team training effect fade out, or is it counteracted by an opposing effect 
Table 2 Primary and secondary procedural outcome, relative risk

\begin{tabular}{|c|c|c|c|c|c|c|}
\hline & $\begin{array}{l}\text { Period } 0 \\
\mathrm{n}(\%)\end{array}$ & $\begin{array}{l}\text { Period } \\
0 \text { vs } 1[\mathrm{Cl}]\end{array}$ & $\begin{array}{l}\text { Period } 1 \\
\mathrm{n}(\%)\end{array}$ & $\begin{array}{l}\text { Period } \\
1 \text { vs } 2[\mathrm{Cl}]\end{array}$ & $\begin{array}{l}\text { Period } 2 \\
n(\%)\end{array}$ & $\begin{array}{l}\text { Period } \\
0 \text { vs } 2[\mathrm{Cl}] \\
\end{array}$ \\
\hline Failed extraction & $61(19)$ & & $28(8)$ & & $37(10)$ & \\
\hline RR, crude & & $\begin{array}{l}0.41 \\
{[0.27-0.62]} \\
p<0.001\end{array}$ & & $\begin{array}{l}1.3 \\
\text { NS }\end{array}$ & & $\begin{array}{l}0.53 \\
{[0.36-0.77} \\
p 0.001\end{array}$ \\
\hline $\mathrm{RR}_{\mathrm{a}}$ & & $\begin{array}{l}0.48 \\
{[0.26-0.87]} \\
p 0.016\end{array}$ & & NA & & $\begin{array}{l}0.59 \\
\text { NS }\end{array}$ \\
\hline $\begin{array}{l}\text { Complicated } \\
\text { extraction* }\end{array}$ & $55(17)$ & & $77(22)$ & & $39(11)$ & \\
\hline RR, crude & & $1.3 \mathrm{NS}$ & & $\begin{array}{l}0.47 \\
{[0.33-0.68]} \\
p<0.001\end{array}$ & & $\begin{array}{l}0.61 \\
{[0.42-0.90} \\
\text { p } 0.012\end{array}$ \\
\hline $\mathrm{RR}_{\mathrm{a}}$ & & NA & & $\begin{array}{l}0.42 \\
{[0.23-0.76]} \\
p 0.004\end{array}$ & & $\begin{array}{l}0.53 \\
\text { NS }\end{array}$ \\
\hline
\end{tabular}

Poisson regression. P-value below 0.017 (0.05/3 for Bonferroni correction) considered significant

$\mathrm{RR}_{\mathrm{a}}$ : adjusted by year of birth

*duration $>15$ min or number of pulls $>6$, or cup detachment $>1$

of traction force measurement? Or was there no true exposure effect between period 0 and period I, but merely a regression towards the mean and residual confounding? Interestingly, the proportion of low and mid cavity extractions increased during the study period, a situation with known increased risk for failure compared to outlet vacuum extraction. Unfortunately, the design does not allow for a separate analysis of the two exposure effects.
An increased emergency cesarean section rate as the price to pay for fewer (failed) extractions may seem intuitive, but this observation is not necessarily a direct effect of fewer vacuum extractions; emergency cesarean sections performed at a fully dilated cervix without a prior attempt at vacuum or forceps did not increase significantly during the studied time periods. This may indicate that obstetricians did not become more prone to

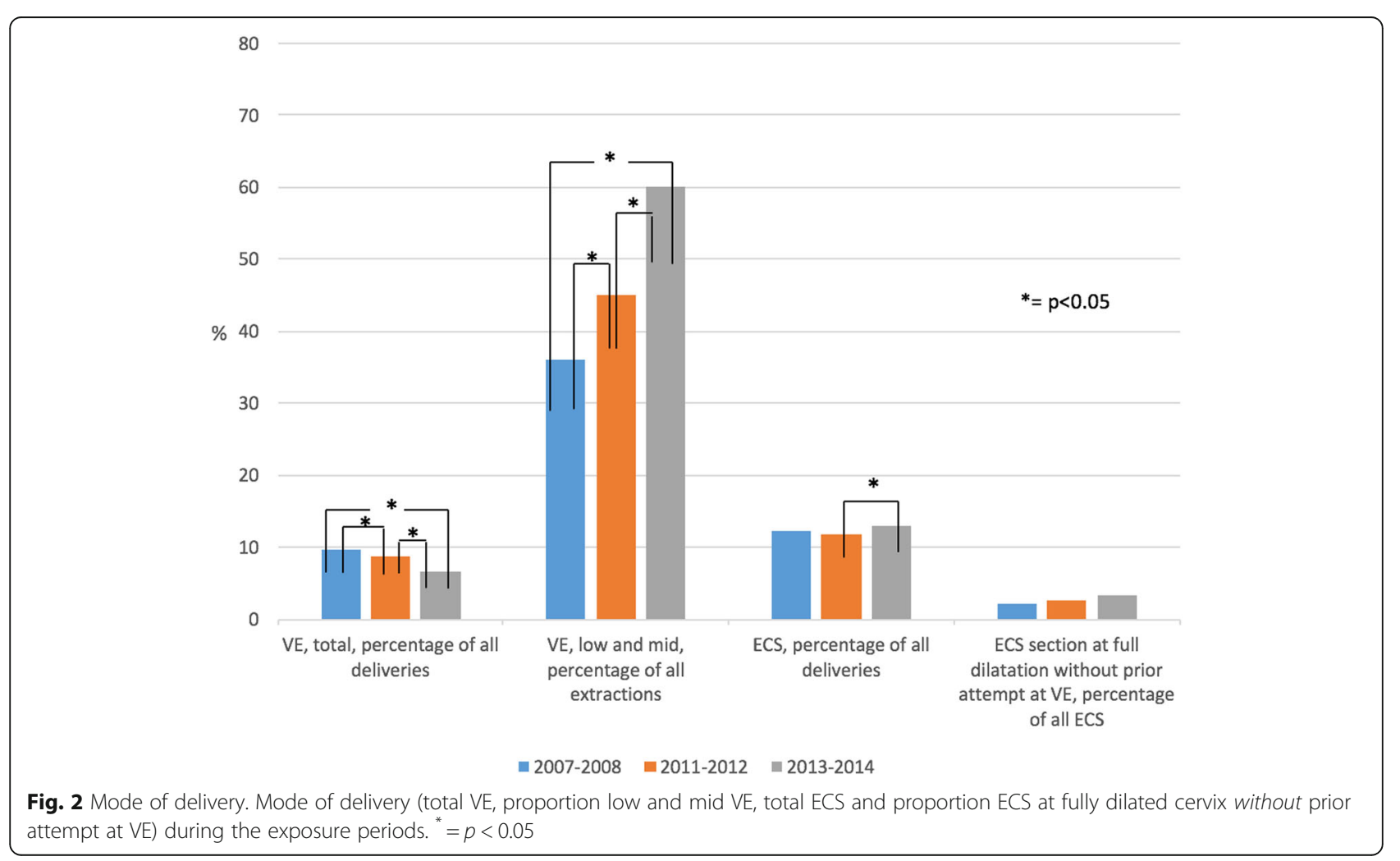


choose cesarean section instead of vacuum, since the increase in emergency cesarean section rates were not predominantly in the category that could have undergone an attempted extraction. A recent Swedish hospital based study on approximately 8000 nulliparous women further support the notion that obstetric care enhancing efforts can lead to decreased vaginal instrumental delivery without a simultaneous increase in emergency cesarean section [13]. The monitoring of period 2 seems to have inferred an increased adherence to clinical guidelines, measured as a decreased risk of complicated extraction (prolonged duration or more than six pulls or more than one cup detachment). These factors are recurring in some guidelines $[1,3]$, whilst others stress the lack of evidence regarding concrete limitations [2]. Studies investigating the clinical effect of restricting extractions regarding duration, number of pulls and cup detachment show inconsistent results [6, 26, 27], and the relative importance of avoiding failed extractions vs adhering to guidelines therefore remains an open question.

\section{Strengths and limitations}

The major limitation of the pre-post test study design is arguably the restricted possibility of making causal inference: this design, also known as quasi experimental design, has inherent bias regarding comparison groups due to either a total absence of parallel control group (as in this particular study) or non-randomized control group, as well as differences due to the passage of time. Some confounding is arguably avoided by adjusting for year of birth, but there will still be residual confounding of which we do not know the magnitude. This might include known confounders where we lack data, such as policy changes, staff turnover and proportion of resident vs consultant doctors, as well as variables that we have overlooked. We are also aware of the specific problem of regression towards the mean; normally, an intervention is introduced because of an identified problem, and one can therefore expect a non-representatively extreme value of crucial variables at the study start. With a $19 \%$ failure rate among mid and low extractions in period 0 , the pre-post comparison is likely to over-estimate the effect size. To overcome this, a prospective study with pre-specified evaluation protocol and a parallel control group would be a suggested alternative method.

In period 1 , the imprecision of the components mediating the training effect to the decreased failure rate makes it difficult to interpret the results. Reflecting upon the clinical characteristics of Table 1 , the effect was clearly not mediated by a population of taller and less dystocic women, nor smaller infants or an increased use of oxytocin. In this material, therefore, we lack an obvious explanatory model. One strength regarding exposure is that the team training set-up contained most of the active components of effective team training identified previously in a review [14].

In exposure period 2, it is reasonable to think that monitoring traction force would make obstetricians more cautious and prone to adhere to guidelines when selecting cases for and performing vacuum extraction. In a monitoring situation, a more cautious selection of candidates for vacuum extraction might be recognized as a decreasing proportion of mid cavity station and non-occiput anterior position extractions from period 1 to period 2, but this is not confirmed by our results. However, the decreased risk of complicated extraction in period 2 might support a possible effect of awareness during monitoring. In a population based study on failed extractions [19], the authors argue that a small increase in failed extractions during a period of educational efforts might actually be an effect of sensible obstetric decisions to consciously convert a difficult extraction instead of employing maximum traction force. Our finding of a partly negative association between the rate of failed extractions and rate of complicated extractions makes a possible illustration of this phenomenon.

\section{Conclusion}

Some of the decrease in failed extractions is likely an effect of active educational effort. The possible monitoring effect of the traction force measurements in period 2 did not seem to deter obstetricians from mid station extractions, but the monitoring may have led to an increased adherence to clinical guidelines.

Despite the limitations to inferring causality in this study design, the results support that alterations in clinical practice should be accompanied by clinical team education.

\section{Additional files}

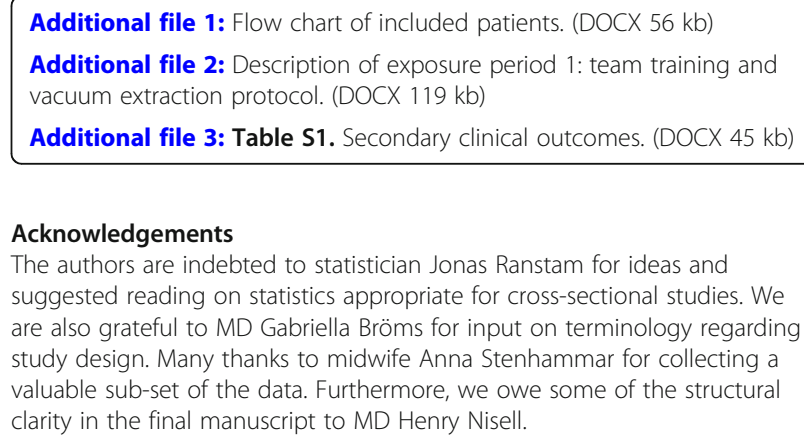
suggested reading on statistics appropriate for cross-sectional studies. We are also grateful to MD Gabriella Bröms for input on terminology regarding study design. Many thanks to midwife Anna Stenhammar for collecting a valuable sub-set of the data. Furthermore, we owe some of the structural clarity in the final manuscript to MD Henry Nisell.

\section{Author contributions}

GA: project development, data collection and analysis, manuscript revision. RG-E: project development, data collection and analysis, manuscript revision. MW: Project development, data management and planned analysis, manuscript revision. KP: project development, data collection and analysis, 
manuscript drafting. All authors read and approved the final version of the manuscript.

\section{Funding}

All authors are supported by grants provided by the Stockholm County Council (ALF 553176 and junior clinical research grant project 108182). The founder had no role in design of the study, collection, analysis, interpretation of data, or in writing the manuscript.

\section{Availability of data and materials}

The data are originating from personal data, and can therefore not be publically available. However, the data sets can be provided upon reasonable request by the corresponding author (KP).

\section{Ethics approval and consent to participate}

Ethical approval has been obtained by the Swedish Ethical Review Board. 2014/1860-31.

2017/1411-32

\section{Consent for publication}

Not applicable.

\section{Competing interests}

None of the authors have any competing interests to declare.

\section{Publisher's Note}

Springer Nature remains neutral with regard to jurisdictional claims in published maps and institutional affiliations.

Received: 19 December 2018 Accepted: 22 March 2019

Published online: 29 March 2019

\section{References}

1. Royal College of Obstetricians and Gynaecologists. Operative vaginal delivery. Green-top Guideline No 26. 2011;26:2011.

2. ACOG Practice Bulletin No. 154: operative vaginal delivery. Obstet Gynecol 2015;126(5):e56-e65.

3. Vladic-Stjernholm Y WM AM, Amer-Whålin I. Instrumental delivery by vacuum extraction: advice for clinical recommendations (in Swedish): Löf; 2017 [Available from: https://lof.se/wp-content/uploads/Instrumentellförlossning-med-sugklocka.pdf.

4. O'Mahony F, Settatree R, Platt C, Johanson R. Review of singleton fetal and neonatal deaths associated with cranial trauma and cephalic delivery during a national intrapartum-related confidential enquiry. BJOG : an international journal of obstetrics and gynaecology. 2005;112(5):619-26.

5. Ekeus C, Hogberg U, Norman M. Vacuum assisted birth and risk for cerebral complications in term newborn infants: a population-based cohort study. BMC pregnancy and childbirth. 2014;14:36

6. Simonson C, Barlow P, Dehennin N, Sphel M, Toppet V, Murillo D, et al. Neonatal complications of vacuum-assisted delivery. Obstet Gynecol. 2007; 109(3):626-33.

7. Doumouchtsis SK, Arulkumaran S. Head injuries after instrumental vaginal deliveries. Curr Opin Obstet Gynecol. 2006;18(2):129-34.

8. Martin JA, Hamilton BE, Osterman MJ, Curtin SC, Matthews TJ. Births: final data for 2013. Natl Vital Stat Rep. 2015;64(1):1-65.

9. Nystrom ME, Westerlund A, Hoog E, Millde-Luthander C, Hogberg U, Grunewald $C$. Healthcare system intervention for prevention of birth injuries - process evaluation of self-assessment, peer review, feedback and agreement for change. BMC Health Serv Res. 2012;12:274

10. Millde Luthander C, Kallen K, Nystrom ME, Hogberg U, Hakansson S, Harenstam KP, et al. Results from the National Perinatal Patient Safety Program in Sweden: the challenge of evaluation. Acta Obstet Gynecol Scand. 2016:95(5):596-603.

11. Laine K, Skjeldestad FE, Sandvik L, Staff AC. Incidence of obstetric anal sphincter injuries after training to protect the perineum: cohort study. BMJ Open. 2012;2(5)

12. Brown LD, Permezel $M$, Holberton JR, Whitehead $C L$. Neonatal outcomes after introduction of a national intrapartum fetal surveillance education program: a retrospective cohort study. J Matern Fetal Neonatal Med. 2017 30(15):1777-81.
13. Blomberg M. Avoiding the first cesarean section--results of structured organizational and cultural changes. Acta Obstet Gynecol Scand. 2016; 95(5):580-6.

14. Siassakos D, Crofts JF, Winter C, Weiner CP, Draycott TJ. The active components of effective training in obstetric emergencies. BJOG : an international journal of obstetrics and gynaecology. 2009;116(8):1028-32.

15. Murphy DJ, Macleod M, Bahl R, Strachan B. A cohort study of maternal and neonatal morbidity in relation to use of sequential instruments at operative vaginal delivery. Eur J Obstet Gynecol Reprod Biol. 2011;156(1):41-5.

16. Gardella C, Taylor M, Benedetti T, Hitti J, Critchlow C. The effect of sequential use of vacuum and forceps for assisted vaginal delivery on neonatal and maternal outcomes. Am J Obstet Gynecol. 2001;185(4): 896-902.

17. Edgar DC, Baskett TF, Young DC, O'Connell CM, Fanning CA. Neonatal outcome following failed kiwi OmniCup vacuum extraction. J Obstet Gynaecol Can. 2012;34(7):620-5.

18. Al-Kadri H, Sabr Y, Al-Saif S, Abulaimoun B, Ba'Aqeel H, Saleh A. Failed individual and sequential instrumental vaginal delivery: contributing risk factors and maternal-neonatal complications. Acta Obstet Gynecol Scand. 2003:82(7):642-8.

19. Ahlberg $M$, Norman $M$, Hjelmstedt $A$, Ekeus $C$. Risk factors for failed vacuum extraction and associated complications in term newborn infants: a population-based cohort study. J Matern Fetal Neonatal Med. 2016;29(10): 1646-51.

20. Towner D, Castro MA, Eby-Wilkens E, Gilbert WM. Effect of mode of delivery in nulliparous women on neonatal intracranial injury. N Engl J Med. 1999; 341(23):1709-14.

21. Pettersson K, Ajne J, Yousaf K, Sturm D, Westgren M, Ajne G. Traction force during vacuum extraction: a prospective observational study. BJOG : an international journal of obstetrics and gynaecology. 2015;122(13):1809-16.

22. McCambridge J, Witton J, Elbourne DR. Systematic review of the Hawthorne effect: new concepts are needed to study research participation effects. J Clin Epidemiol. 2014:67(3):267-77.

23. Pettersson K, Yousaf K, Ranstam J, Westgren M, Ajne G. Predictive value of traction force measurement in vacuum extraction: development of a multivariate prognostic model. PLoS One. 2017;12(3):e0171938.

24. Lee J, Tan CS, Chia KS. A practical guide for multivariate analysis of dichotomous outcomes. Ann Acad Med Singap. 2009;38(8):714-9.

25. Barros AJ, Hirakata VN. Alternatives for logistic regression in cross-sectional studies: an empirical comparison of models that directly estimate the prevalence ratio. BMC Med Res Methodol. 2003;3:21.

26. Ghidini A, Stewart D, Pezzullo JC, Locatelli A. Neonatal complications in vacuum-assisted vaginal delivery: are they associated with number of pulls, cup detachments, and duration of vacuum application? Arch Gynecol Obstet. 2017;295(1):67-73.

27. Ekeus C, Wrangsell K, Penttinen S, Aberg K. Neonatal complications among 596 infants delivered by vacuum extraction (in relation to characteristics of the extraction). J Matern Fetal Neonatal Med. 2018;31(18):2402-8.

Ready to submit your research? Choose BMC and benefit from:

- fast, convenient online submission

- thorough peer review by experienced researchers in your field

- rapid publication on acceptance

- support for research data, including large and complex data types

- gold Open Access which fosters wider collaboration and increased citations

- maximum visibility for your research: over $100 \mathrm{M}$ website views per year

At BMC, research is always in progress.

Learn more biomedcentral.com/submissions 